http://jmscr.igmpublication.org/home/ ISSN (e)-2347-176x ISSN (p) 2455-0450 crossref DOI: https://dx.doi.org/10.18535/jmscr/v8i6.77

\title{
Epidermodysplasia Verruciformis: A Case Report
}

Authors

\author{
Dilbag Singh ${ }^{1}$, Ravinder Singh ${ }^{2 *}$, Shikha Verma ${ }^{3}$ \\ ${ }^{1}$ Senior Resident Dermatology JLNGMC Chamba HP \\ ${ }^{2}$ Medical Officer Dermatology Dr RKGMC Hamirpur HP \\ ${ }^{3}$ Senior Resident Pediatrics DR RPGMC Kangra at Tanda \\ *Corresponding Author \\ Ravinder Singh
}

\begin{abstract}
Epidermodysplasia verruciformis (EV) is a rare disorder that is usually transmitted in an autosomal recessive manner, caused by human papillomavirus. It has tendency for malignant transformation, especially squamous cell carcinoma. These patients show a defect in cell-mediated immunity specific toward the causative HPVs that lead to lifelong disease. The defect is usually inherited as autosomal recessive trait and presents clinically with plane warts, pityriasis versicolor like lesions and reddish verrucous plaques. Here we present a case of 15 year boy with complaints of asymptomatic hypopigmented macular lesions and flat wart-like lesions over the face, neck, hands and forearms. Histopathology was suggestive of EV. He was given oral zinc, sunscreen and advised regular follow up.
\end{abstract}

\section{Introduction}

Epidermodysplasia verruciformis (EDV) is an uncommon cutaneous disorder characterized by persistent infection with human papillomavirus (HPV) and a combination of flat wart like lesions and hypopigmented macules. These lesions are mainly located on the face, neck, and extremities ${ }^{[1]}$. $\mathrm{EV}$ is most commonly inherited in an autosomal recessive manner although sporadic, sex linked, and autosomal dominant inheritance has been described $^{[2]}$. It has a potential for malignant transformation, especially squamous cell carcinoma.

\section{Case Report}

A 15 years old boy came to the skin outpatient department with complaints of asymptomatic lesions over body since the age of 8 years, which were gradually increasing in number. There were no other systemic complaints. Examination revealed, multiple hypopigmented maculopapular lesions, few of them coalescing to form patches, were present over the neck, retroauricular region, front of chest and multiple hyperkeratotic verrucous papules over dorsum of both hands (figure). Scalp, oral cavity, genitals, and nails were normal. Systemic examination was unremarkable. A punch biopsy was taken from the lesion over the back of hands suggestive of plane warts without atypia. Other laboratory investigations including complete blood counts, serum biochemistry, HIV serology and urinalysis were essentially normal.

Above mentioned features were suggestive of EDV. The patient was advised strict photoprotection and was started on oral zinc, topical $0.025 \%$ retinoic acid. 


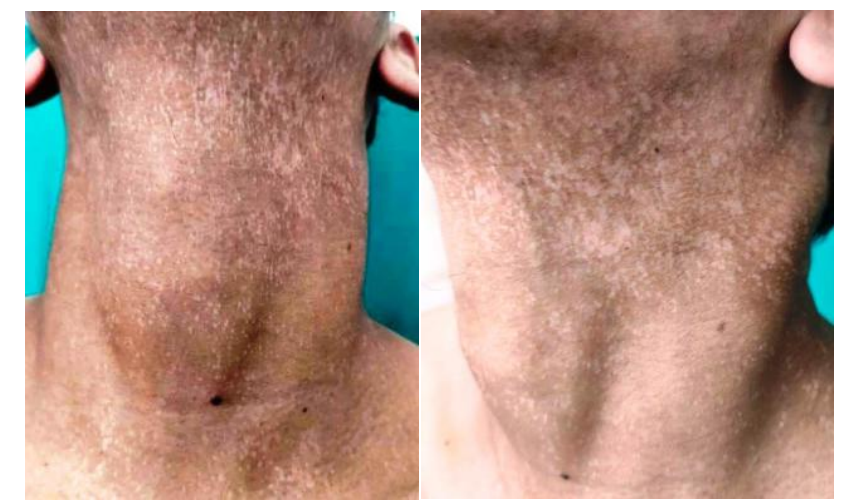

Figure: 1, 2 Involvement of front and side of neck multiple hypopigmented macules coalescing into patches

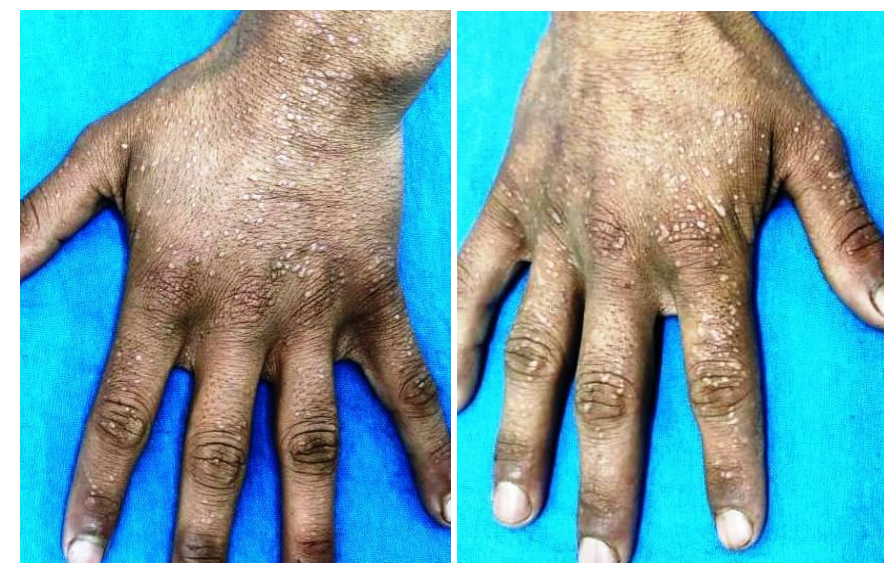

Figure: 3, 4 Involvement of dorsum of hands with multiple verrucous papules

\section{Discussion}

Epidermodysplasia verruciformis (EV) is a rare genodermatosis characterized by wide spread and persistent infection with human papilloma viruses (HPVs), presenting clinically with characteristic combination of pityriasis versicolor-like lesions, reddish verruca-like and seborrheic keratosis-like plaques with a potential for malignant transformation. This malignant transformation largely depends upon the oncogenic potential of the infecting virus. To date more $100 \mathrm{HPV}$ types have been identified but mostly HPV 3, 5, 8, 9, 10, 12, 14, $17,19-25,28$ and 29 are related to $\mathrm{EV}^{[2,3]}$ and more than one HPV type may be present simultaneously in the same patient. HPV 5 and 8 are the main types incriminated for the malignant transformation in about $25 \%$ of EV patients ${ }^{[3,4]}$. Deficiency in cell mediated immunity specific to HPV results in a natural inhibition of cytotoxic mechanisms against infection by HPV, leading to the development of skin lesions in classical EV. This susceptibility of human keratinocytes to the HPV in EV is usually inherited through an autosomal recessive gene and pathogenic mutations have been detected in EVER1/TMC6 and EVER2/TMC8 genes mapped to a locus on chromosome 17q 25 that encodes integral membrane proteins of unknown significance in the endoplasmic reticulum ${ }^{[5]}$. Immune status of the patient, viral phenotype and sun exposure influences progression and evolution of skin lesions to malignancy, particularly squamous cell carcinoma ${ }^{[6]}$ Important carcinogenic cofactors include ultraviolet (UV) B and X-rays. There are some reports of acquired EV associated with immunosuppression states such as renal transplantation, graft versus host disease, systemic lupus erythematosus, and HIV infection ${ }^{[7]}$. EV occurs in all races, without sex predilection and is most common during childhood, puberty with age of onset between 5 and 8 years. It can present with three types of lesions, pityriasis versicolor like, verruca plana like, seborrheic keratosis like in isolation or in combination ${ }^{[8]}$. Initially the lesions are localized, hypochromic scaly patches on the face and neck, similar to tinea versicolor. Over time, they increase in number and tend to evolve to papules resembling flat warts from pink to brownish, sized a few millimeters, with a smooth surface. Transformation of EV lesions into SCC occurs in 30\%-70\% cases and may require over 20 years for such conversion. Actinic damage is perhaps the pathogenic mechanism involved in malignant transformation of lesions over extremities and forehead ${ }^{[9]}$. EV should be suspected in a setting of numerous verrucous lesions recalcitrant to appropriate therapy. Biopsy is performed for early detection of premalignant and malignant lesions and for the identification of EV associated $\mathrm{HPV}^{[4]}$. EV has pathognomonic histological features. The keratin layer is loose with a basket weave like appearance. The most characteristic findings are the presence of clear cells in the granular and spinous layers with occasional enlarged, hyperchromatic, atypical nuclei. The nucleoplasm is clear, and keratohyaline granules of various sizes and shapes 
are present. The premalignant lesions display features similar to actinic keratoses with prominent atypical, dyskeratotic cells. Currently, there is no specific or effective treatment for EV. Management includes preventive measures such as genetic counselling, photoprotection, and monitoring of symptoms for proper identification of premalignant and malignant lesions. Sunscreens are recommended to avoid direct exposure to $\mathrm{UV}$ radiation since constant exposure tends to increase the risk of malignancy. Oral zinc, due to its immunomodulatory effects, had been used by many authors either alone as well as in combination therapy ${ }^{[2]}$. Acitretin $0.5-1 \mathrm{mg} /$ day too has been effective and is perhaps the drug of choice currently $^{[10]}$. Nonsurgical approaches include topical imiquimod, 5- fluorouracil, Vitamin D3, cimetidine, systemic retinoids and interferon which are only palliative and improve quality of life, need large controlled studies for recommending their lifelong use.

\section{References}

1. Yoshida R, Kato T, Kawase M, Honda M, Mitsuishi T. Two sisters reveal autosomal recessive inheritance of epidermodysplasia verruciformis: A case report. BMC Dermatol 2014;14:12.

2. Sharma S, Barman KD, Sarkar R, Manjhi M, Garg VK. Efficacy of oral zinc therapy in epidermodysplasia verruciformis with squamous cell carcinoma. Indian Dermatol Online J 2014;5:55- 8.

3. Ostrow RS, Manias D, Mitchell AJ, Stawowy L, Faras AJ. Epidermodysplasia verruciformis. A case with primary lymphatic dysplasia, depressed cellmediated immunity, and Bowen's disease containing human papilloma 16 DNA. Arch Dermatol 1987;123:1511-6.

4. Pfister H. Human papilloma viruses and impaired immunity vs epidermodysplasia verruciformis. Arch Dermatol 1987;123: 1469-70.
5. Ramoz N, Rueda LA, Bouadjar B, Montoya LS, Orth G, Favre M. Mutations in two adjacent novel genes are associated with epidermodysplasia verruciformis. Nat Genet 2002;32:579- 81.

6. Castro- Pérez GA, Sorin I, Bravo AI, Mazzuoccolo LD. Acquired epidermodysplasia verruciformis in a patient with congenital HIV infection. Actas Dermosifiliogr2013;104:731- 3

7. Rodríguez L, Contreras R, Di Martino Ortiz B, de Lezcano LB. Acquired epidermodysplasia verruciformis in an HIV positive child. Report of a case. Our Dermatol Online 2015;6:32- 5.

8. Roncalli de Oliveira W, Neto CF, Rady PL, Tyring SK. Seborrheic Keratosis- like lesions in patients with epidermodysplasia verruciformis. J Dermatol 2003;30:48- 53.

9. Gül U, Kiliç A, Gönül M, Cakmak SK, Bayis SS. Clinical aspects of epidermodysplasia verruciformis and review of the literature. Int J Dermatol 2007;46: 1069- 7.

10. Vohra S, Sharma NL, Shanker V, Mahajan VK, Jindal N. Autosomal dominant epidermodysplasia verruciformis: A clinicotherapeutic experience in two cases. Indian J Dermatol Venereol Leprol 2010;76:557- 61.

11. García-Río I, Garcia-F-Villalta MJ, Daudén E, Fraga J, García-Díez A. Epidermodysplasia verruciformis-like lesions in a patient with systemic lupus erythematosus. Acta Derm Venereol 2003;83:229-30. 